\title{
Delayed voluntary exercise does not enhance cognitive performance after hippocampal injury: an investigation of differentially distributed exercise protocols
}

\author{
Elise Wogensen', Marie Gajhede Gram', Jens Bak Sommer², Christina Rytter Vilsen', Jesper Mogensen', Hana Malá1,* \\ ${ }^{1}$ The Unit for Cognitive Neuroscience, Department of Psychology, University of Copenhagen, Copenhagen, Denmark \\ 2Department of Drug Design and Pharmacology, University of Copenhagen, Copenhagen, Denmark
}

Voluntary exercise has previously been shown to enhance cognitive recovery after acquired brain injury (ABI). The present study evaluated effects of two differentially distributed protocols of delayed, voluntary exercise on cognitive recovery using an allocentric place learning task in an 8-arm radial maze. Fifty-four Wistar rats were subjected to either bilateral transection of the fimbria-fornix (FF) or to sham surgery. Twenty-one days postinjury, the animals started exercising in running wheels either for 14 consecutive days (FF/exercise daily [ExD], sham/ExD) or every other day for 14 days (FF/exercise every second day [ExS], sham/
ExS). Additional groups were given no exercise treatment (FF/not exercise [NE], sham/NE). Regardless of how exercise was distributed, we found no cognitively enhancing effects of exercise in the brain injured animals. Design and protocol factors possibly affecting the efficacy of post-ABI exercise are discussed.

Keywords: Voluntary exercise, Animal model, Cognitive recovery, Spatial learning, Brain injury, Hippocampus.

\section{INTRODUCTION}

There is a growing interest in the effects of exercise on cognitive abilities and health. Clinical research has focused on cognitive effects of physical activity in healthy populations (Khan and Hillman, 2014; Smith et al., 2010), as well as in people with damage to the central nervous system (Beier et al., 2014; Öhman et al., 2014).

Cognitive impairment after acquired brain injury (ABI) is common and can compromise many mental abilities such as memory, attention, processing speed and executive function. Cognitive rehabilitation tends to focus on psychoeducation, training of specific cognitive domains, compensational strategies, and metacognitive awareness (Cicerone et al., 2011; Greenwald and Rigg, 2009). Physical exercise therapy on the other hand is a commonly employed rehabilitation tool to promote motor recovery after ABI (Greenwald and Rigg, 2009; Marshall et al., 2007). However,

there are strong indications that exercise also improves cognitive functioning after ABI. Most data stems from preclinical models of brain injury (El-Tamawy et al., 2014; Grealy et al., 1999). Animal model-based research shows that exercise can promote cognitive recovery under certain circumstances, but findings are not consistent (Wogensen et al., 2015). Many animal model based studies have focused on the cognitive effects of exercise initiated early after injury ( $0-4$ days postinjury). However, it is rare that brain injured patients presenting solely with cognitive problems receive exercise training as part of their rehabilitation plan in the early postinjury stages - if at all. Furthermore, cognitive dysfunctions can be difficult to diagnose in the early postinjury phases. To investigate whether exercise performed during later recovery phases can also promote cognitive recovery, we applied a delayed exercise paradigm (exercise starting 21 days postinjury) in the present study.

Presently, it is still unknown how, when and how much one should exercise to gain optimal effects on cognitive recovery. A

*Corresponding author: Hana Malá (D) http://orcid.org/0000-0002-2910-372X The Unit for Cognitive Neuroscience, Department of Psychology, University of Copenhagen Oester Farimagsgade 2A, DK-1353 Copenhagen K, Denmark Tel: +45-35324875, Fax: +45-35324802, E-mail: hana.mala@psy.ku.dk Received: July 14, 2016 / Accepted: September 5, 2016

This is an Open Access article distributed under the terms of the Creative Commons Attribution Non-Commercial License (http://creativecommons.org/licenses/by-nc/4.0/) which permits unrestricted non-commercial use, distribution, and reproduction in any medium, provided the original work is properly cited. 
scarcely investigated, but potentially important, factor is the temporal distribution of exercise - that is, whether one should exercise daily or whether there should be resting day(s) between exercise sessions. In most animal model studies, animals exercise every day (Wogensen et al., 2015). However, in a study by Cechetti et al. (2012) animals exposed to bilateral common carotid artery occlusion, were exercised three times a week for 12 weeks. This administration schedule improved learning, reference memory and working memory recovery in the brain injured animals. To our knowledge there are no studies directly comparing the cognitive effects of distributed versus continuous (daily) administered exercise after brain injury. As many patients in outpatient rehabilitation settings do not receive daily exercise training, it is relevant to address if there are differential effects of daily versus more distributed exercise. In this study, we therefore investigated the effects of voluntary exercise administered either daily or every other day for a total period of 14 days.

While $\mathrm{ABI}$ can cause tissue damage in many cerebral areas, hippocampal atrophy is common in both animal models and clinical studies of traumatic brain injury (Ariza et al., 2006). Related structures, such as the fornix, also show degeneration due to brain trauma (Tate and Bigler, 2000), and this process has been shown to continue into the later postinjury stages (Adnan et al., 2013). It is well-established that the hippocampus and its surrounding structures are involved in learning and memory processing (Opitz, 2014) as well as spatial representation (Moser et al., 2008). In the present study, we employed a partial model of brain injury, namely bilateral transection of the fimbria-fornix, which causes retrograde degeneration of the medial and dorsolateral septal nuclei and compromises the functionality of the hippocampus (Ginsberg and Martin, 1998; Li et al., 2005). This procedure is known to induce substantial learning and memory deficits in rats in a variety of cognitive tasks (Malá et al., 2005; Mogensen et al., 2004; Mogensen et al., 2007). Using this model of mechanical brain injury, we presently address the clinically important issue whether differentially distributed exercise protocols may be able to promote posttraumatic cognitive recovery when administered at a relatively late posttraumatic time point.

\section{MATERIALS AND METHODS}

\section{Subjects and experimental groups}

Fifty-four experimentally naïve, male, Wistar rats weighing approx. $300 \mathrm{~g}$ at the beginning of the experiment served as experimental subjects. The animals were pair-housed in makrolon type
3 cages with elevated lids (allowing rearing in the cage) under controlled temperature $\left(22^{\circ} \mathrm{C} \pm 2^{\circ} \mathrm{C}\right)$ and humidity $(50 \% \pm 5 \%)$. A reversed 12-hr light/dark cycle was maintained in the animal quarters (lights on at 7:00 p.m.) and all experimental procedures took place during the animals' dark phase. During the exercise period the animals were given commercial rat chow ad libitum. During behavioral procedures in the maze, the animals were fed once daily after training/testing and maintained at approximately $85 \%$ of their ad libitum body weights. Water was always available ad libitum.

The animals were randomly divided into six experimental groups:

(1) Sham surgery group not subjected to exercise $(n=10$, Sham/ $\mathrm{NE})$

(2) Sham surgery group subjected to exercise daily ( $\mathrm{n}=8$, Sham/ ExD)

(3) Sham surgery group subjected to exercise every second day ( $\mathrm{n}=8$, Sham/ExS)

(4) Fimbria-fornix transected group not subjected to exercise $(\mathrm{n}=12, \mathrm{FF} / \mathrm{NE})$

(5) Fimbria-fornix transected group subjected to exercise daily $(\mathrm{n}=8, \mathrm{FF} / \mathrm{ExD})$

(6) Fimbria-fornix transected group subjected to exercise every second day $(\mathrm{n}=8, \mathrm{FF} / \mathrm{ExS})$

The experiments were carried out in accordance with the guidelines of the Danish Animal Experimentation Act and the European Council Directive of 22 September 2010 (2010/63/EU). All efforts were made to minimize the number of animals used and their suffering according to the principle of the three Rs.

\section{Voluntary exercise in running wheels}

The voluntary exercise took place in ENV-042 activity wheels with attached modular holding cages (MED Associates Inc., St Albans, VT, USA). The holding cage-from which the animal could freely enter and exit the running wheel—was $17.8 \mathrm{~cm}$ in height, $16 \mathrm{~cm}$ wide and $20.3 \mathrm{~cm}$ in depth and equipped with bedding and unlimited access to water. The wheel was $35.6 \mathrm{~cm}$ in diameter and $11 \mathrm{~cm}$ in width with a free wheeling drag of approximately $12 \mathrm{~g}$. The number of wheel revolutions per every 5 min was recorded by computer software (Ellegaard Systems A/S, Faaborg, Denmark). The mean number of revolutions was calculated for each period of $6 \mathrm{hr} /$ day.

Rats had access to the running wheel apparatus during their dark period. Preoperatively - and before introduction to the 8-arm radial maze - the animals were habituated to the running wheels 
for $2 \mathrm{hr} /$ day for three consecutive days. Postoperatively, the animals in the exercise groups were given access to the wheels either $6 \mathrm{hr}$ daily for 14 consecutive days (a total of 14 exercise sessions) or $6 \mathrm{hr}$ every second day for 14 days (a total of seven exercise sessions). All animals were weighed daily throughout the exercise period.

\section{Allocentric place learning task in 8-arm radial maze}

The behavioral procedures have been described elsewhere (Malá et al., 2005). All cognitive training and testing was performed in an open, darkly colored, 8-arm radial maze with $2.7 \mathrm{~cm}$ high walls and $9.2 \mathrm{~cm}$ wide corridors. The circular center of the maze had a diameter of $50 \mathrm{~cm}$, from which eight arms, all $60 \mathrm{~cm}$ long, spread out with equal spacing. At the end of each arm a circular food well (diameter, $4.8 \mathrm{~cm}$; depth, $2.3 \mathrm{~cm}$ ) contained reinforcements in the form of four 45-mg food pellets (Precision Food Pellets, Campden Instruments, Leics, UK). The maze was placed in the center of a well-lit room in which a multitude of two- and three-dimensional visual cues were available.

All training and testing of animals in the 8-arm radial maze were performed by trained experimenters who were blinded regarding the group affiliations of the animals. Preoperatively, the animals were habituated to the 8-arm radial maze for 2 days (16 min per day) and then shaped to promptly enter one of the arms of the maze for 19 consecutive days (15 trials a day). On the 43rd postoperative day, the animals resumed the behavioral procedures in the 8-arm radial maze. During the first three of these postoperative sessions, the animals were reshaped. On the fourth postoperative session, training and testing in an allocentric place learning task was initiated. All animals were given 15 trials per day for 28 consecutive days. One arm (defined according to its spatial location within the experimental room) was chosen as the goal arm. The remaining seven arms served as start arms and were selected in a randomized order. To limit the animals' use of intramazial cues for the task solution, the maze was rotated daily before the beginning of testing. When entering the goal arm, the animal was allowed to reach the food well and consume four reinforcement pellets. If an incorrect arm was entered, the animal was picked up before reaching the food well. After each trial the animal was removed from the maze and put in a holding cage for approx. one minute. From each session, two parameters were recorded: 'number of total errors' (i.e., the number of times per session the animal did not choose the goal arm) and 'number of distal errors' (i.e., the number of times per session the animal chose neither the goal arm nor the two arms adjacent to the goal arm). Additionally, the parameters 'summed errors' (i.e., the summated
Table 1. Schematic overview of the behavioral procedures

\begin{tabular}{|c|c|c|c|}
\hline Experimental phase & Duration & $\begin{array}{l}\text { Experimental } \\
\text { group }\end{array}$ & Feeding \\
\hline $\begin{array}{l}\text { Habituation to } \\
\text { exercise apparatus }\end{array}$ & 3 Days & All exercise groups & Ad libitum \\
\hline Habituation to maze & 2 Days & All & Food deprivation \\
\hline Shaping & 19 Days & All & Food deprivation \\
\hline Surgery & $30 \mathrm{Min}$ & All & Ad libitum \\
\hline Postsurgery break & 21 Days & All & Ad libitum \\
\hline Exercise & $\begin{array}{l}14 \text { Days (either } \\
\text { daily or bi-daily) }\end{array}$ & $\begin{array}{l}\text { Sham or FF: ExD, } \\
\text { ExS }\end{array}$ & Ad libitum \\
\hline Reshaping & 3 Days & All & Food deprivation \\
\hline Place learning task & 28 Days & All & Food deprivation \\
\hline
\end{tabular}

FF, fimbria-fornix; ExD, exercise daily; ExS, exercise every second day.

number of total errors across all 28 sessions) and 'summed distal errors' (i.e., the summated number of distal errors across all 28 sessions) were calculated for each animal across all sessions. See Table 1 for an overview of the behavioral procedures.

\section{Surgery}

Surgery (which lasted approximately $30 \mathrm{~min}$ per animal) was performed with the aid of a surgical microscope under clean conditions. The animals were anesthetized with medetomidine (Dexdomitor, $0.37 \mathrm{mg} / \mathrm{kg}$ ) and ketamine (Ketaminol, $62 \mathrm{mg} / \mathrm{kg}$ ) and subsequently given atropine $(0.3 \mathrm{mg} / \mathrm{kg})$ - all via intraperitoneally injections. The animals' eyes were lubricated with a protective salve (Neutral Ophtha) to prevent drying. The incision site was shaved, iodized and a topical analgesic (lidocain) was injected into the skin.

Bilateral transections of the fimbria-fornix were performed stereotaxically as described in detail elsewhere (Mogensen et al., 2004). To summarize, a hole was drilled into the skull $1.1 \mathrm{~mm}$ posteriorly to the bregma and $1.2 \mathrm{~mm}$ laterally to the sagittal suture, and a guiding cannula containing a wire-knife was lowered to a position $3.2 \mathrm{~mm}$ ventrally to the dura and extended $1.6 \mathrm{~mm}$ laterally. The wire-knife was further lowered to a position $5.0 \mathrm{~mm}$ ventrally to the dura and left for $1 \mathrm{~min}$. The extended wire-knife was then returned to a position $3.2 \mathrm{~mm}$ ventrally to the dura. The knife was retracted into the guiding cannula and the instrument rotated $180^{\circ}$. Following extension, the wire-knife was again lowered to a position $5.0 \mathrm{~mm}$ ventrally to the dura and left for $1 \mathrm{~min}$. The extended wire-knife was then returned to the position 3.2 $\mathrm{mm}$ ventrally to the dura. After retraction into the guiding cannula, the cannula was withdrawn from the brain tissue. The procedures were performed in both hemispheres. The sham operated 
animals were anaesthetized and their skulls exposed, however, the brain remained intact.

Prior to surgery, animals were habituated for 3 days to ingest Nutella (Ferrero, Alba, Italy), a hazelnut/chocolate spread. Immediately following surgery, all animals were given analgesic treatment with buprenorphine (Temgesic injection fluid, $0.03 \mathrm{mg} / \mathrm{kg}$ ) and sterile saline ( $2 \mathrm{~mL}$ per animal) via subcutaneous injections. The animals were put in clean cages and kept under heating lamps until emerging from the anaesthesia. Analgesic treatment (Temgesic sublingual tablets, $0.03 \mathrm{mg} / \mathrm{kg}$, crushed and mixed in Nutella) was administered twice daily and continued as long as needed. After cessation of the analgesic treatment, animals were given pure Nutella once a day for 2 days to avoid a sudden weight loss. Commercial rat chow soaked in water was provided to ease feeding during recovery and optimize water intake.

\section{Histology}

Upon finishing the behavioral testing, the animals were deeply anesthetized and transcardially perfused with $10 \%$ sucrose followed by a $4 \%$ buffered formaldehyde in saline solution. The brains were removed and stored at $4{ }^{\circ} \mathrm{C}$ in $4 \%$ buffered formaldehyde in saline solution. Brain sections were cut horizontally on a vibratome at $50 \mu \mathrm{m}$ and examined under a microscope (Leica DMD 108, Leica Microsystems, Wetzlar, Germany), to verify the locus of the lesion and to quantify the size of lesion. The proportion of fimbria-fornix fibres that remained intact was quantified using the microscope associated software. The transection was examined through its entire depth and measurements regarding two levels representing the dorsal and ventral edge of the FF fiber bundle were recorded: level 1 (dorsal: interaural, 6.40; bregma, -3.60) and level 2 (ventral: interaural, 5.72; bregma, -4.28) (Paxinos and Watson, 1986). The obtained values were averaged in the sham and lesioned animals, respectively, and percentage of the intact fibers in the lesioned animals was calculated relative to animals subjected to control sham surgery. The values regarding lesion sizes in the lesioned groups were subsequently analysed statistically.

\section{Statistics analysis}

All statistical analyses were performed using IBM SPSS Statistics ver. 22.0 (IBM Co., Armonk, NY, USA).

The size of lesion in the three fimbria-fornix transected groups (FF/NE, FF/ExD, and FF/ExS) was estimated and compared using independent samples Kruskal-Wallis nonparametric test. Body weights during exercise were analysed by two-way analysis of variance (ANOVA) to identify potential group differences on the first and last day of exercise administration. Paired-sample t-tests were used to compare within-group body weight changes between the first and last day of exercise.

Distances (m) run in the exercise wheels were analysed using a mixed design, repeated measures ANOVA over 14 exercise sessions (Sham/ExD and FF/ExD) and seven exercise sessions (Sham/ ExS and FF/ExS). Total between-group mean running distances were analysed by two-way ANOVA.

Overall acquisition of the place learning task ('summed total errors' and 'summed distal errors') was analysed using two-way ANOVAs. To identify lesion effects and acquisition over time ('number of total errors' and 'number of distal errors'), mixed design, repeated-measures ANOVAs were performed using the means obtained within each of seven groups of four sessions (sessions $1-4,5-8,9-12,13-16,17-20,21-24$, and 25-28). In case of violated sphericity, Greenhouse-Geisser correction was applied.

In order to identify the specific effects of the exercise procedures in the lesioned and control operated (sham) groups, separate mixed design, repeated-measures ANOVAs (using similar session intervals as above) were executed for sham and fimbria-fornix transected groups, respectively. All significances are two-tailed. Significance level is defined as $P<0.05$.

\section{RESULTS}

\section{Anatomy}

The histological examination revealed that in all of the lesioned animals, the fimbria-fornix rostral to the dorsal hippocampus was almost completely transected - only a minor portion of the fibres remained intact. There was no significant difference in the percentage of transected fibers in the three fimbria-fornix transected groups (FF/ExD, $\mathrm{FF} / \mathrm{ExS}$, and $\mathrm{FF} / \mathrm{NE})(P=0.340)$. In all animals, the fimbria and dorsal fornix were transected at the level of the ventral hippocampal commissure (anteroposterior distance to bregma, $-1.30 \mathrm{~mm}$ to $-1.40 \mathrm{~mm}$ ). In a few animals, the damage extended ventrally into the subfornical organ, dorsally into the ventral part of corpus callosum, and laterally into the dorsomedial neostriatum. Fig. 1 schematically summarizes the extent of the lesion and shows that it did not differ across experimental groups.

\section{Body weights during exercise}

A two-way ANOVA of body weights on the first day of exercise revealed no effect of surgery $(F[1,48]=0.210, P=0.649)$, exercise $(F[2,48]=0.372, P=0.691)$ or surgery $\times$ exercise $(F[2,48]=0.318$, $P=0.729$ ). This was also the case on the last day of exercise (sur- 


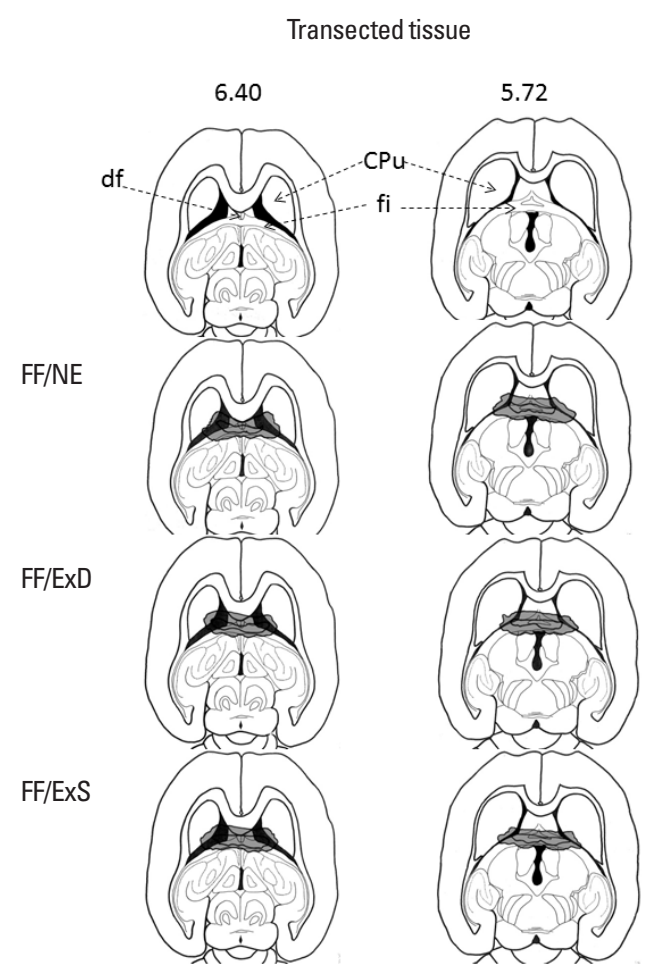

Fig. 1. Schematic diagram of the fimbria-fornix transections. The gray area indicates the lesioned area. The outer line corresponds to area lesioned in at least one animal. The inner line corresponds to the area transected in all animals. Illustrations of the three groups that were subjected to the fimbria-fornix transection: the group not subjected to exercise (FF/NE), the group subjected to exercise daily (FF/ExD), and the group subjected to exercise every second day (FF/ExS) (for more information see 'MATERIALS AND METHODS' and 'RESULTS' sections). The diagrams show horizontal levels 6.40 and 5.72 in front of the interaural line (Paxinos and Watson, 1986). df, dorsal fornix; fi, fimbria; $\mathrm{CPu}$, caudate putamen.

gery: $F[1,48]=0.022, P=0.883$; exercise: $F[2,48]=2.441, P=$ 0.098 ; surgery $\times$ exercise: $F[2,48]=0.763, P=0.472$ ).

Paired-sample $t$-tests comparing first and last day body weights within each individual group revealed weight increases from start to end in Sham/NE $(P<0.001) ;$ Sham/ExS $(P<0.005) ;$ FF/NE $(P<$ $0.001)$, and $\mathrm{FF} / \operatorname{ExS}(P<0.01)$, and no weight differences in Sham/ ExD and $\mathrm{FF} / \operatorname{ExD}(P=0.422$ and $P=0.344$, respectively) (Fig. 2).

\section{Running wheel exercise}

Mean running distances across exercise sessions were $264.7 \mathrm{~m}$ (Sham/ExD); $367.6 \mathrm{~m}$ (FF/ExD); $366.9 \mathrm{~m}$ (Sham/ExS); and 408.0 $\mathrm{m}(\mathrm{FF} / \mathrm{ExS})$.

A mixed design, repeated measures ANOVA of running distances of the groups allowed access to running wheels for 14 consecutive days (Sham/ExD and FF/ExD) showed a significant effect of time $(F[3.607,50.496]=2.892, P<0.05)$, i.e., increased run-

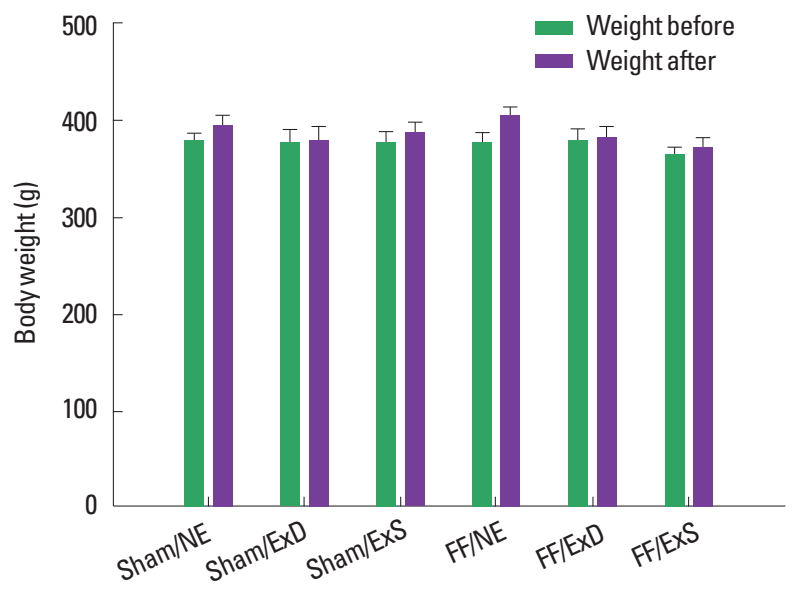

Fig. 2. Body weights (g) on the first day (green bars) and last day (purple bars) of exercise. All values are given as means with standard error of the mean. Sham/NE, sham surgery group not subjected to exercise; Sham/ExD, sham surgery group subjected to exercise daily; Sham/ExS, sham surgery group subjected to exercise every second day; FF/NE, fimbria-fornix transected group not subjected to exercise; FF/ExD, fimbria-fornix transected group subjected to exercise daily; FF/ExS, fimbria-fornix transected group subjected to exercise every second day.

ning over time in both groups. No time $\times$ surgery effect $(F[3.607$, $50.496]=1.258, P=0.299$ ) was found (Fig. 3A).

A mixed design, repeated measures ANOVA of running distances in the groups running every other day (Sham/ExS and FF/ExS) showed no effects of time $(F[2.934,41.070]=1.996, P=0.131)$ or time $\mathrm{x}$ surgery $(F[2.934,41.070]=0.509, P=0.674)$ (Fig. 3B).

Subsequent two-way ANOVA of mean total distances comparing all exercise groups showed no effect of either surgery $(F[1,28]=$ $0.893, P=0.353)$, exercise $(F[1,28]=0.912, P=0.348)$, or surgery $\times$ exercise $(F[1,28]=0.167, P=0.686)$ (Fig. $3 C$ ).

\section{Behavior}

A two-way ANOVA investigating 'summed total errors' revealed a significant effect of surgery $(F[1,48]=130.970, P<0.001)$, indicating significantly fewer errors in the sham-groups compared to the FF-groups (Fig. 4A). There was no effect of exercise ( $F[2$, $48]=1.177, P=0.317)$ or surgery $\times$ exercise $(F[2,48]=1.710$, $P=0.192)$. The same analysis using 'summed distal errors' as the dependent variable produced a similar picture with a clear effect of surgery, but no other significant effects (surgery: $F[1,48]=87.171$, $P<0.001$; exercise: $F[2,48]=0.639, P=0.532$; surgery $\times$ exercise: $F[2,48]=1.419, P=0.252$ ) (Fig. 4B). This illustrates, that the lesioned animals performed significantly worse on both investigated parameters than the sham-operated animals regardless of exercise treatment. 

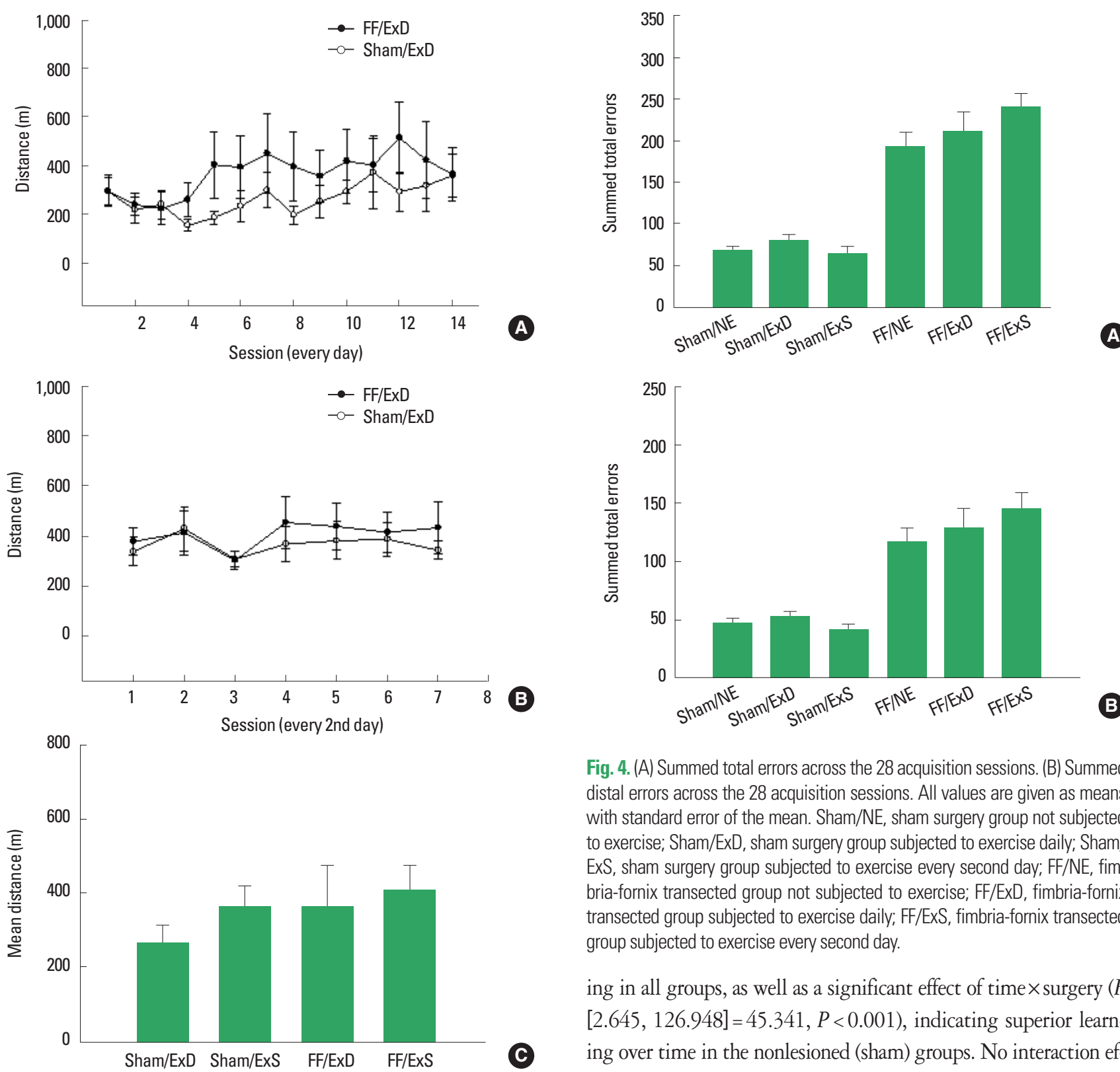

Fig. 4. (A) Summed total errors across the 28 acquisition sessions. (B) Summed distal errors across the 28 acquisition sessions. All values are given as means with standard error of the mean. Sham/NE, sham surgery group not subjected to exercise; Sham/ExD, sham surgery group subjected to exercise daily; Sham/ ExS, sham surgery group subjected to exercise every second day; FF/NE, fimbria-fornix transected group not subjected to exercise; FF/ExD, fimbria-fornix transected group subjected to exercise daily; FF/ExS, fimbria-fornix transected group subjected to exercise every second day.

ing in all groups, as well as a significant effect of time $\times$ surgery $(F$ $[2.645,126.948]=45.341, P<0.001)$, indicating superior learning over time in the nonlesioned (sham) groups. No interaction effects of timex exercise $(F[5.289,126.948]=0.987, P=0.431)$ or time $\times$ exercise $\times$ surgery $(\mathrm{F}[5.289,126.948]=1.133, P=0.347)$

Fig. 3. (A) Running distances ( $\mathrm{m}$ ) of groups exercised daily for a total of 14 sessions. Open circle: Sham/ExD; black circle: FF/ExD. (B) Running distances (m) of groups exercised every second day for a total of 7 sessions. Open circle: Sham/ExS; black circle: FF/ExS. (C) Total running distances (m) of all exercised groups. Values are given as means with standard error of the mean. Sham/ ExD, sham surgery group subjected to exercise daily; Sham/ExS, sham surgery group subjected to exercise every second day; FF/ExD, fimbria-fornix transected group subjected to exercise daily; FF/ExS, fimbria-fornix transected group subjected to exercise every second day.

A mixed design, repeated measures ANOVA of 'number of total errors' of every four-session interval revealed a significant effect of time $(F[2.645,126.948]=310.791, P<0.001)$, indicating learnwere found. Between-subjects analysis showed a clear lesion effect $(F[1,48]=130.970, P<0.001)$, however, no effect of exercise ( $F[2$, $48]=1.177, P=0.317)$ or surgery $\times$ exercise interaction effect $(F[2$, $48]=1.710, P=0.192)$. Using the same analysis with 'number of distal errors' as the dependent variable revealed a significant effect of time $(F[2.884,138.435]=259.074, P<0.001)$ and time $\times$ surgery $(F[2.884,138.435]=32.987, P<0.001)$, but no interaction effects regarding timexexercise $(F[5.768,138.435]=1.029, P=$ $0.408)$ or time $\times$ surgery $\times$ exercise $(F[5.768,138.435]=0.413, P=$ $0.863)$. Between-subjects analysis demonstrated an effect of surgery 

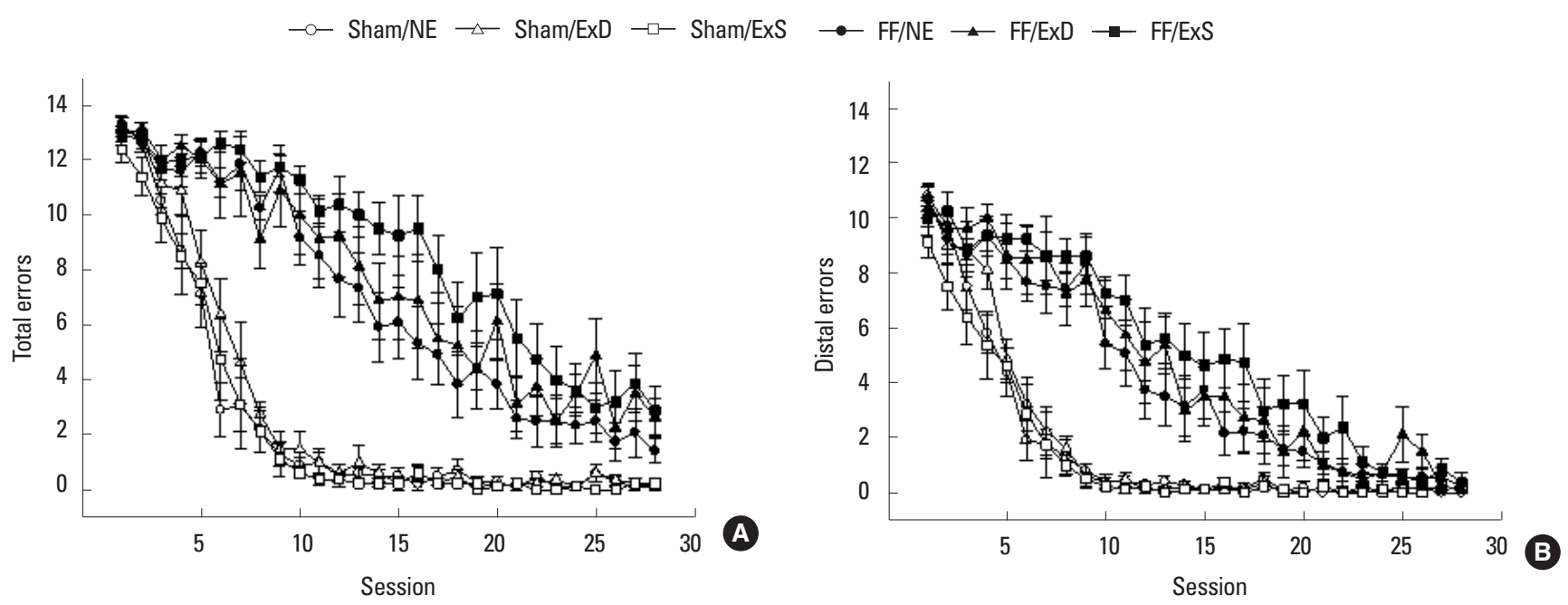

Fig. 5. (A) Number of total errors during acquisition of the allocentric place learning task. (B) Number of distal errors during acquisition of the allocentric place learning task. Open circle: Sham/NE, open triangle: Sham/ExD, open square: Sham/ExS, black circle: FF/NE, black triangle: FF/ExD, black square: FF/ExS. All values are given as means with standard error of the mean. Sham/NE, sham surgery group not subjected to exercise; Sham/ExD, sham surgery group subjected to exercise daily; Sham/ExS, sham surgery group subjected to exercise every second day; FF/NE, fimbria-fornix transected group not subjected to exercise; FF/ExD, fimbria-fornix transected group subjected to exercise daily; FF/ExS, fimbria-fornix transected group subjected to exercise every second day.

$(F[1,48]=87.171, P<0.001)$, but no effects of exercise or surgery $\times$ exercise $(F[2,48]=0.639, P=0.532$ and $F[2,48]=1.419, P=$ 0.252 , respectively).

In order to investigate possible separate effects of exercise in the intact and brain injured animals, subsequent mixed design, repeated measures ANOVAs (regarding 'number of total errors' and 'number of distal errors') were performed in the lesioned and sham groups, respectively. Analysing 'number of total errors' in the three lesioned groups (FF/NE, FF/ExD, and FF/ExS) revealed a time effect $(F[2.593,64.819]=118.532, P<0.001)$, but no time $\mathrm{x}$ exercise effect $(F[5.185,64.819]=1.150, P=0.344)$. Between-subject analysis revealed no significant differences between exercise groups $(F[2,25]=1.537 ; P=0.235)$, indicating that the exercise intervention did not affect task acquisition. Regarding 'number of distal errors' the same analysis also showed an effect of time $(F[2.791,69.768]=110.537, P<0.001)$, but not of time $\times$ exercise $(F[5.581,69.768]=0.498, P=0.796)$. There were no significant between-groups differences $(F[2,25]=1.056$; $P=0.363$ ), again indicating no effects of the exercise treatment on the number of distal errors in the lesioned groups.

Analysis of the performance of the sham-operated groups (Sham/ $\mathrm{NE}$, Sham/ExD, and Sham/ExS) showed a significant effect of time $(F[1.540,35.416]=346.740, P<0.001)$ regarding 'number of total errors'. There was neither a significant timex exercise effect $(F$ $[3.080,35.416]=0.959, P=0.425)$ nor significant effect of the between-subjects analysis $(F[2,23]=1.676, P=0.209)$. Regarding 'number of distal errors', the overall pattern was the same, showing an effect of time $(F[1.865,42.892]=308.957, P<0.001)$, but no interaction effect $(F[3.730,42.892]=1.733, P=0.164)$ or between-subjects effect $(F[2,23]=1.711, P=0.203)$.

\section{DISCUSSION}

In the current study we found that voluntary exercise initiated 21 days after bilateral transection of the fimbria-fornix did not enhance cognitive recovery - neither when the exercise was carried out daily for 14 days nor when it was administered every other day for 14 days (Fig. 5). This result is surprising, as other studies have reported cognitively enhancing effects of voluntary exercise paradigms administered in the late stages after brain injury (Gram et al., 2016; Piao et al., 2013; Winocur et al., 2012; Wong-Goodrich et al., 2010). Such results have, however, not been obtained in all cases (Clark et al., 2008).

Several factors limit our knowledge regarding the effects of exercise on cognitive recovery in animals subjected to ABI. Firstly, animal model-based research on the subject in general - and studies investigating effects of late exercise (i.e., three weeks or more after injury) in particular - is scarce and methodologically diverse. Secondly, animal studies on effects of voluntary exercise starting in the early stages after $\mathrm{ABI}$ (i.e., during the first three postinjury weeks) show a somewhat mixed picture. Some studies find positive effects on cognitive recovery, some report no changes and some 
find detrimental effects (Wogensen et al., 2015). Research on the effects of forced exercise paradigms after ABI generally find positive effects of early initiated exercise (Itoh et al., 2011, Kim et al., 2010, Sim et al., 2004). Given this picture, one may be tempted to conclude that exercise most effectively promotes cognitive recovery when administered in the early postinjury stages. However, the available data regarding effects of late exercise on cognitive recovery after brain injury is limited to only a few studies (Wogensen et al., 2015). A delayed administration of exercise most closely resembles the clinical practice, where exercise is sometimes used as a supportive intervention in comprehensive rehabilitation programs in the postacute phase after ABI. More research is therefore needed to elucidate the effects of delayed exercise on parameters of cognitive recovery.

Out of the limited number of studies investigating the cognitive recovery effects of exercise administered at later time points after ABI, most use radiation exposure models (Clark et al., 2008; Winocur et al., 2012; Wong-Goodrich et al., 2010). It is presently unclear whether outcomes of exercise intervention in irradiation models can be directly compared to other models of ABI. Cancer studies have shown that therapeutic doses of irradiation can cause neurotoxic effects - especially affecting hippocampal neurogenesis, cell proliferation, differentiation and apoptosis (Dietrich et al., 2008; Monje, 2008). Stroke or traumatic brain injuries generally produce more widespread and diffuse cerebral pathologies (Bramlett and Dietrich, 2004), although hippocampal atrophy is a common finding in human survivors of traumatic brain injury (Ariza et al., 2006; Green et al., 2014; Tomaiuolo et al., 2004). The injury model used in the present study (fimbria-fornix transection) is a focal mechanical injury specifically targeting hippocampal functioning. On one hand, there is a basis for comparison with irradiation-induced brain injury models as both models specifically affect hippocampal processes. On the other hand, the injury infliction as well as the processes known to be affected by the transection differ from those of irradiation-associated injury. As part of the septo-hippocampal pathway, the fimbria-fornix relays important cholinergic input to the hippocampus, which is significantly reduced by the transection (Ayala-Grosso et al., 2004). The transection has also been shown to affect hippocampal long-term potentiation (Li et al., 2005). The fact that the positive effects of exercise reported by some of the irradiation studies (for references see above) are not replicated in our study may therefore be attributable to differences in model pathologies.

Other potentially important factors could also have contributed to the lack of exercise effects in the current study: running dis- tance as well as intensity and duration of exercise. The most widely used measure of activity in voluntary exercise paradigms is the average distance run over sessions. In the current study, the lesioned animals ran $368 \mathrm{~m}$ (FF/ExD) and $408 \mathrm{~m}$ (FF/ExS) on average per exercise session. These distances are comparable to distances found in other studies showing recovery enhancing effects after late voluntary exercise (Gram et al., 2016; Piao et al., 2013; Wong-Goodrich et al., 2010). Positive effects of post-ABI voluntary exercise on cognitive recovery have been found in animals running between approximately $100 \mathrm{~m}$ and (more than) $8 \mathrm{~km}$ a day (Wogensen et al., 2015), showing that both short and long distances can promote recovery. In our study we see no such recovery promoting effects. This could mean that either the animals in our study ran too short a distance to promote recovery or the average running distances are not a reliable measures of exercise efficiency. Moreover, it is known that different mice and rat stocks/ strains exhibit different patterns of spontaneous activity in the running wheel with some types running longer distances than others (Coletti et al., 2013; Johnson and Mitchell, 2003; Lightfoot et al., 2004). For instance, investigating spontaneous running wheel activity in 14 different mouse strains, Lightfoot et al. (2004) found significant variations not only in running distances, but also in durations and velocity of running. This suggests that the genetic background of animals can influence the pattern and intensity with which they exercise and that the choice of rodent strain/stock might therefore influence recovery outcome in voluntary running paradigms. This might also contribute to the heterogeneous results reported by studies in this field. As none of the studies reporting cognition enhancing outcomes after voluntary exercise in brain injured rats used the Wistar stock, one may speculate whether the animals in our study had a sufficient spontaneous activity level to promote cognitive recovery.

Additionally, voluntary exercise paradigms do not allow experimental control of running intensity. Studies using forced exercise paradigms indicate that intensity plays a role for the efficacy of exercise, with low-to-moderate intensity leading to enhanced cognitive recovery (Shen et al., 2013; Shih et al., 2013; Shimada et al., 2013). However, this is not a homogenous finding, as more high intensity exercise has also been reported to induce positive effects on cognitive recovery (Cechetti et al., 2012; Itoh et al., 2011), while low intensity exercise in some cases has been without effect (Chen et al., 2006). These findings suggest that exercise intensity is an important factor for promotion of recovery, and that the lack of intensity control associated with voluntary exercise could be an intrinsic weakness of the paradigm. Furthermore, ex- 

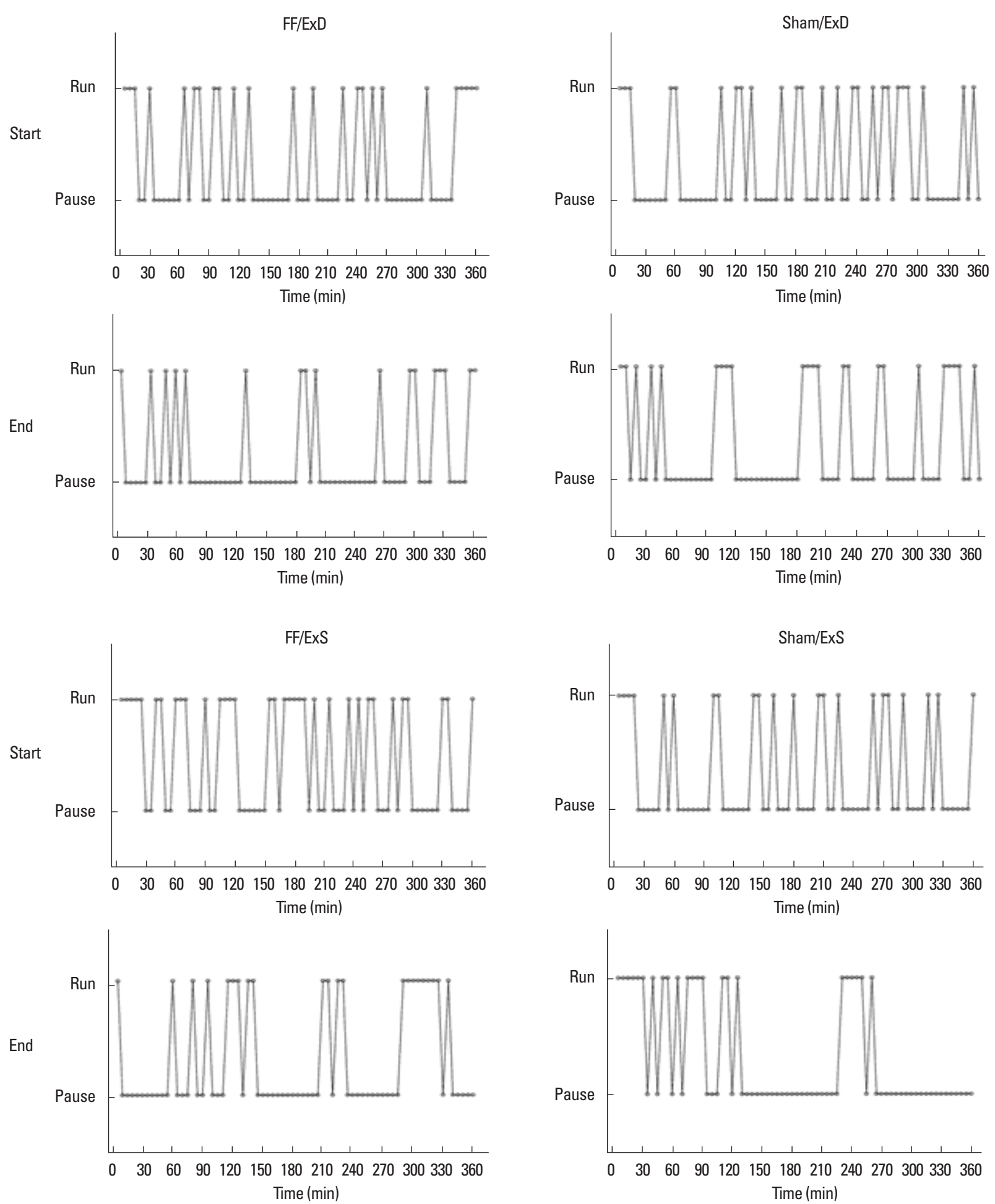

Fig. 6. Running and pausing pattern examples of animals in the four exercise groups (FF/ExD, FF/ExS, Sham/ExD, Sham/ExS) at the beginning and end of the exercise period. Measurements were obtained every $5 \mathrm{~min}$ (indicated by dots) for $6 \mathrm{hr}$. Pausing was defined as $<5$ wheel rotations per every 5 min measuring point, running was defined as $\geq 5.1$ wheel rotations per every 5 min measuring point. Sham/ExD, sham surgery group subjected to exercise daily; Sham/ExS, sham surgery group subjected to exercise every second day; FF/ExD, fimbria-fornix transected group subjected to exercise daily; FF/ExS, fimbria-fornix transected group subjected to exercise every second day. 
ercise perseverance and pauses in activity can also influence exercise efficacy. Most forced exercise paradigms demand uninterrupted running at a specific pace, often for $30 \mathrm{~min}$ (Wogensen et al., 2015). However, such running continuity may not be obtained when using voluntary exercise. Ploughman et al. (2007) found that animals in nonmotorized activity wheels ran and stopped frequently. Running pattern samplings from the present study has revealed a similar picture with intermittent periods of running and pausing (Fig. 6). Furthermore, the samplings indicate that the length of the pauses increases during the exercise period (i.e., from day 1 through day 7 to day 14). Daily running distances, however, do not decrease (Fig. 3A, B). Thus, it appears that the pattern of running changes during the exercise period (Fig. 6). As a consequence of this changed running pattern, the animals in our set-up may not have reached the level of intensity or running continuity necessary to promote cognitive recovery.

A related question has to do with the duration of the intervention - both per session and the number of exercise sessions. Many voluntary exercise paradigms allow 24-hr access to running wheels in the home cages (Griesbach et al., 2009; Winocur et al., 2012). However, this often entails that the animals are single housed. Single housing has been shown to decrease neurogenesis and the levels of brain-derived neurotrophic factor and increase stress-susceptibility in intact animals (Berry et al., 2012; Leasure and Decker, 2009; Stranahan et al., 2006). In the present study we allowed the animals $6 \mathrm{hr}$ of access to the exercise apparatus (during their active phase) and then returned the animal to its home cage with its cage mate. To our knowledge, it is currently unresolved whether and how single housing affects cognitive recovery in brain injured animals. Future research should also apply designs that control for social deprivation as a confounding factor in the study of exercise effects on cognitive recovery. Moreover, total duration of the exercise may also play a role. Effects on cognitive recovery have been found in voluntary paradigms administering exercise for 1 week up to approximately 6-week duration (Wogensen et al., 2015). The 14 days of exercise administered in this study should therefore fall within the potentially therapeutic range. However, as already mentioned, other variables, such as injury, timing and running intensity, are likely to be responsible for the lack of therapeutic effects in the current set-up.

Finally, it is unknown whether the applied intervention was too general to promote recovery or whether recovery of the applied cognitive task would require a more task-specific training. Several studies in healthy animals have suggested that task-specific (i.e., learning based) limb training is more effective in producing plas- tic changes in the motor cortex than a more general activation through mere limb use (Kleim et al., 1998). Using rats subjected to cerebral ischemia, Biernaskie and Corbett (2001) showed that a combination of general enriched environment and specific skilled motor training resulted in better motor recovery and increased neuronal plasticity in the motor cortex than animals kept in standard housing conditions. This opens the question of whether exercise would be more effective as a postinjury intervention if it has a concomitant task learning aspect. Further research is needed to elucidate if more generally activating exercise protocols (i.e., using more than one limb) with a concomitant task-specific learning element could promote recovery—including cognitive recoveryafter brain injury.

Thus, we are still in the early stages of elucidating the effects of late voluntary exercise on cognitive recovery after ABI. While research into the cognitive effects of exercise in both preclinical and clinical populations show promise, research methods and results are still highly diverse. Factors such as type of injury, animal species, timing and dose of the exercise intervention appear to be central for the effects on cognitive outcome. Future research in this area would benefit from more methodologically homogenous studies further investigating these parameters.

\section{CONFLICTS OF INTEREST}

The authors have nothing to disclose.

\section{ACKNOWLEDGMENTS}

The present study was supported by a grant from the Danish Council for Independent Research. The authors wish to thank Martin Blumensaadt Bach, Varvara Bashkirova, Jennifer Cooper, Patricia Fernández, Siri Mehlsen, Laura Obel, Jeppe Olsen, and Nikolaj Daugaard Sørensen for their assistance with the behavioral testing.

\section{REFERENCES}

Adnan A, Crawley A, Mikulis D, Moscovitch M, Colella B, Green R. Moderate-severe traumatic brain injury causes delayed loss of white matter integrity: evidence of fornix deterioration in the chronic stage of injury. Brain Inj 2013;27:1415-1422.

Ariza M, Serra-Grabulosa JM, Junqué C, Ramírez B, Mataró M, Poca A, Bargalló N, Sahuquillo J. Hippocampal head atrophy after traumatic brain injury. Neuropsychologia 2006;44:1956-1961. 
Ayala-Grosso C, Tam J, Xanthoudakis S, Bureau Y, Roy S, Nicholson DW, Robertson GS. Effects of fimbria-fornix transection on calpain and choline acetyl transferase activities in the septohippocampal pathway. Neuroscience 2004;126:927-940.

Beier M, Bombardier CH, Hartoonian N, Motl RW, Kraft GH. Improved physical fitness correlates with improved cognition in multiple sclerosis. Arch Phys Med Rehabil 2014;95:1328-1334.

Berry A, Bellisario V, Capoccia S, Tirassa P, Calza A, Alleva E, Cirulli F. Social deprivation stress is a triggering factor for the emergence of anxiety- and depression-like behaviours and leads to reduced brain BDNF levels in C57BL/6J mice. Psychoneuroendocrinology 2012;37: 762-772.

Biernaskie J, Corbett D. Enriched rehabilitative training promotes improved forelimb motor function and enhanced dendritic growth after focal ischemic injury. J Neurosci 2001;21:5272-5280.

Bramlett HM, Dietrich WD. Pathophysiology of cerebral ischemia and brain trauma: similarities and differences. J Cereb Blood Flow Metab 2004;24:133-150.

Cechetti F, Worm PV, Elsner VR, Bertoldi K, Sanches E, Ben J, Siqueira IR, Netto CA. Forced treadmill exercise prevents oxidative stress and memory deficits following chronic cerebral hypoperfusion in the rat. Neurobiol Learn Mem 2012;97:90-96.

Chen L, Gong S, Shan LD, Xu WP, Zhang YJ, Guo SY, Hisamitsu T, Yin $\mathrm{QZ}$, Jiang XH. Effects of exercise on neurogenesis in the dentate gyrus and ability of learning and memory after hippocampus lesion in adult rats. Neurosci Bull 2006;22:1-6.

Cicerone KD, Langenbahn DM, Braden C, Malec JF, Kalmar K, Fraas M, Felicetti T, Laatsch L, Harley JP, Bergquist T, Azulay J, Cantor J, Ashman T. Evidence-based cognitive rehabilitation: updated review of the literature from 2003 through 2008. Arch Phys Med Rehabil 2011; 92:519-530.

Clark PJ, Brzezinska WJ, Thomas MW, Ryzhenko NA, Toshkov SA, Rhodes JS. Intact neurogenesis is required for benefits of exercise on spatial memory but not motor performance or contextual fear conditioning in C57BL/6 J mice. Neuroscience 2008;155:1048-1058.

Coletti D, Berardi E, Aulino P, Rossi E, Moresi V, Li Z, Adamo S. Substrains of inbred mice differ in their physical activity as a behavior. ScientificWorldJournal 2013;2013:237260.

Dietrich J, Monje M, Wefel J, Meyers C. Clinical patterns and biological correlates of cognitive dysfunction associated with cancer therapy. Oncologist 2008;13:1285-1295.

El-Tamawy MS, Abd-Allah F, Ahmed SM, Darwish MH, Khalifa HA. Aerobic exercises enhance cognitive functions and brain derived neurotrophic factor in ischemic stroke patients. NeuroRehabilitation 2014; 34:209-213.
Ginsberg SD, Martin LJ. Ultrastructural analysis of the progression of neurodegeneration in the septum following fimbria-fornix transection. Neuroscience 1998;86:1259-1272.

Gram MG, Wogensen E, Moseholm K, Mogensen J, Malá H. Exercise-induced improvement in cognitive performance after fimbria-fornix transection depends on the timing of exercise administration. Brain Res Bull 2016;125:117-126.

Grealy MA, Johnson DA, Rushton SK. Improving cognitive function after brain injury: the use of exercise and virtual reality. Arch Phys Med Rehabil 1999;80:661-667.

Green RE, Colella B, Maller JJ, Bayley M, Glazer J, Mikulis DJ. Scale and pattern of atrophy in the chronic stages of moderate-severe TBI. Front Hum Neurosci 2014;8:67.

Greenwald BD, Rigg JL. Neurorehabilitation in traumatic brain injury: does it make a difference? Mt Sinai J Med 2009;76:182-189.

Griesbach GS, Hovda DA, Gomez-Pinilla F. Exercise-induced improvement in cognitive performance after traumatic brain injury in rats is dependent on BDNF activation. Brain Res 2009;1288:105-115.

Itoh T, Imano M, Nishida S, Tsubaki M, Hashimoto S, Ito A, Satou T. Exercise inhibits neuronal apoptosis and improves cerebral function following rat traumatic brain injury. J Neural Transm (Vienna) 2011;118: 1263-1272.

Johnson RA, Mitchell GS. Exercise-induced changes in hippocampal brain-derived neurotrophic factor and neurotrophin-3: effects of rat strain. Brain Res 2003;983:108-114.

Khan NA, Hillman CH. The relation of childhood physical activity and aerobic fitness to brain function and cognition: a review. Pediatr Exerc Sci 2014;26:138-146.

Kim DH, Ko IG, Kim BK, Kim TW, Kim SE, Shin MS, Kim CJ, Kim H, Kim KM, Baek SS. Treadmill exercise inhibits traumatic brain injury-induced hippocampal apoptosis. Physiol Behav 2010;101:660-665.

Kleim JA, Barbay S, Nudo RJ. Functional reorganization of the rat motor cortex following motor skill learning. J Neurophysiol 1998;80:33213325 .

Leasure JL, Decker L. Social isolation prevents exercise-induced proliferation of hippocampal progenitor cells in female rats. Hippocampus 2009;19:907-912.

Li C, Maier DL, Cross B, Doherty JJ, Christian EP. Fimbria-fornix lesions compromise the induction of long-term potentiation at the Schaffer collateral-CA1 synapse in the rat in vivo. J Neurophysiol 2005;93:30013006.

Lightfoot JT, Turner MJ, Daves M, Vordermark A, Kleeberger SR. Genetic influence on daily wheel running activity level. Physiol Genomics 2004;19:270-276.

Malá H, Alsina CG, Madsen KS, Sibbesen El, Stick H, Mogensen J. Eryth- 
ropoietin improves place learning in an 8-arm radial maze in fimbria-fornix transected rats. Neural Plast 2005;12:329-340.

Marshall S, Teasell R, Bayona N, Lippert C, Chundamala J, Villamere J, Mackie D, Cullen N, Bayley M. Motor impairment rehabilitation post acquired brain injury. Brain Inj 2007;21:133-160.

Mogensen J, Hjortkjaer J, Ibervang KL, Stedal K, Malá H. Prefrontal cortex and hippocampus in posttraumatic functional recovery: spatial delayed alternation by rats subjected to transection of the fimbria-fornix and/or ablation of the prefrontal cortex. Brain Res Bull 2007;73:8695.

Mogensen J, Miskowiak K, Sørensen TA, Lind CT, Olsen NV, Springborg JB, Malá H. Erythropoietin improves place learning in fimbria-fornix-transected rats and modifies the search pattern of normal rats. Pharmacol Biochem Behav 2004;77:381-390.

Monje M. Cranial radiation therapy and damage to hippocampal neurogenesis. Dev Disabil Res Rev 2008;14:238-242.

Moser EI, Kropff E, Moser MB. Place cells, grid cells, and the brain's spatial representation system. Annu Rev Neurosci 2008;31:69-89.

Öhman H, Savikko N, Strandberg TE, Pitkälä KH. Effect of physical exercise on cognitive performance in older adults with mild cognitive impairment or dementia: a systematic review. Dement Geriatr Cogn Disord 2014;38:347-365.

Opitz B. Memory function and the hippocampus. Front Neurol Neurosci 2014;34:51-59.

Paxinos G, Watson C. The rat brain in stereotaxic coordinates. Sydney: Academic Press; 1986.

Piao CS, Stoica BA, Wu J, Sabirzhanov B, Zhao Z, Cabatbat R, Loane DJ, Faden AI. Late exercise reduces neuroinflammation and cognitive dysfunction after traumatic brain injury. Neurobiol Dis 2013;54:252263.

Ploughman M, Granter-Button S, Chernenko G, Attwood Z, Tucker BA, Mearow KM, Corbett D. Exercise intensity influences the temporal profile of growth factors involved in neuronal plasticity following focal ischemia. Brain Res 2007;1150:207-216.

Shen X, Li A, Zhang Y, Dong X, Shan T, Wu Y, Jia J, Hu Y. The effect of different intensities of treadmill exercise on cognitive function deficit following a severe controlled cortical impact in rats. Int J Mol Sci 2013;14:21598-21612.

Shih PC, Yang YR, Wang RY. Effects of exercise intensity on spatial memory performance and hippocampal synaptic plasticity in transient brain ischemic rats. PLoS One 2013;8:e78163.

Shimada H, Hamakawa M, Ishida A, Tamakoshi K, Nakashima H, Ishida $\mathrm{K}$. Low-speed treadmill running exercise improves memory function after transient middle cerebral artery occlusion in rats. Behav Brain Res 2013;243:21-27.

Sim YJ, Kim SS, Kim JY, Shin MS, Kim CJ. Treadmill exercise improves short-term memory by suppressing ischemia-induced apoptosis of neuronal cells in gerbils. Neurosci Lett 2004;372:256-261.

Smith PJ, Blumenthal JA, Hoffman BM, Cooper H, Strauman TA, WelshBohmer K, Browndyke JN, Sherwood A. Aerobic exercise and neurocognitive performance: a meta-analytic review of randomized controlled trials. Psychosom Med 2010;72:239-252.

Stranahan AM, Khalil D, Gould E. Social isolation delays the positive effects of running on adult neurogenesis. Nat Neurosci 2006;9:526-533.

Tate DF, Bigler ED. Fornix and hippocampal atrophy in traumatic brain injury. Learn Mem 2000;7:442-446.

Tomaiuolo F, Carlesimo GA, Di Paola M, Petrides M, Fera F, Bonanni R, Formisano R, Pasqualetti P, Caltagirone C. Gross morphology and morphometric sequelae in the hippocampus, fornix, and corpus callosum of patients with severe non-missile traumatic brain injury without macroscopically detectable lesions: a T1 weighted MRI study. J Neurol Neurosurg Psychiatry 2004;75:1314-1322.

Winocur G, Becker S, Luu P, Rosenzweig S, Wojtowicz JM. Adult hippocampal neurogenesis and memory interference. Behav Brain Res 2012;227:464-469.

Wogensen E, Malá H, Mogensen J. The effects of exercise on cognitive recovery after acquired brain injury in animal models: a systematic review. Neural Plast 2015;2015:830871.

Wong-Goodrich SJ, Pfau ML, Flores CT, Fraser JA, Williams CL, Jones LW. Voluntary running prevents progressive memory decline and increases adult hippocampal neurogenesis and growth factor expression after whole-brain irradiation. Cancer Res 2010;70:9329-9338. 